\title{
Effect of stress-related hormones on host Drosophila fitness depends on endosymbiont Wolbachia genotype
}

\author{
N. Adonyeva*, E. Burdina, N. Gruntenko, I. Rauschenbach \\ Institute of Cytology and Genetics SB RAS, Novosibirsk, Russia \\ *e-mail:nadon@bionet.nsc.ru
}

Key words: heat stress, juvenile hormone, 20-hydroxyecdysone, stress-resistance, Drosophila, Wolbachia

Motivation and Aim: It is the first time the influence of gonadotropins, juvenile hormone $(\mathrm{JH})$ and 20-hydroxyecdysone $(20 \mathrm{E})$ on the heat stress resistance of wildtype $D$. melanogaster females infected with different genotypes of $\alpha$-proteobacteria Wolbachia pipientis was investigated to verify the possibility that endosymbiont promotes adaptation to the host species, affecting the functioning of its protective systems.

Methods and Algorithms: To raise the JH level, we applied 0.1 $\mu$ g JHIII (Sigma-Aldrich), dissolved in $0.5 \mu \mathrm{l}$ acetone, to the abdomen of 4-day females, and $0.5 \mu 1$ pure acetone to control individuals. In order to investigate the effect of level $20 \mathrm{E}$ changes on the resistance to heat stress of $D$. melanogaster females infected with different strains of Wolbachia, on the 4th day after eclosion the flies were placed in viales, the bottom and $1 \mathrm{~cm}$ of the walls of which were covered with filter paper, soaked with $0.5 \mathrm{ml}$ of culture medium containing $0.5 \%$ sucrose, $0.2 \%$ yeast and $60 \mu \mathrm{g} 20 \mathrm{E}$ (Sigma-Aldrich). In the control series in a solution of $20 \mathrm{E}$ was not added. On the 6 th day after eclosion the $\mathrm{JH}$ or $20 \mathrm{E}$-treated and control flies were subjected to thermal stress $\left(38^{\circ} \mathrm{C}, 2\right.$ hours $45 \mathrm{~min}$ ) and 24 hours later the number of surviving flies were counted.

Results: It was found that the experimental increase in the level of the $\mathrm{JH}$ causes a decrease in resistance of 6-day females to heat stress, while the increase in the level of $20 \mathrm{E}$ - its increase, both in uninfected Wolbachia and infected with various strains of bacteria: wMelCS, wMelPop and wMel. However, the intensity of the response differs: in females infected with pathogenic strain wMelPop, the decline in survival at higher levels of the $\mathrm{JH}$ and its increase with increasing levels of $20 \mathrm{E}$ are more pronounced, while in females infected with genotype wMelCS is less pronounced than that in uninfected females and females infected with genotype wMel.

Conclusion: The obtained data indicate that the genotype of $w M e l C S$ causes a decrease, and wMelPop - an increase in the level of stress hormone, dopamine, in female Drosophila, since earlier we showed that an increase in the level of the $\mathrm{JH}$ in mature females increases the level of dopamine, while an increase in the level of 20E reduces it [1], and an increase/decrease in the level of dopamine, in turn, leads to a decrease/ increase in the resistance of female Drosophila to heat stress [2].

Acknowledgements: Supported by the RFBR (16-04-00060) and State budget project No. 0324-2016-0002.

\section{References}

1. Gruntenko N.E., Rauschenbach I.Yu. (2008) Interplay of juvenile hormone, 20-hydroxyecdisone and biogenic amines under normal and stress conditions and its effect on reproduction. J. Insect. Physiol. 56:902-908.

2. Gruntenko N.E. et al. (2004) The effect of mutations altering biogenic amine metabolism in Drosophila on viability and the response to heat stress. Arch. Insect Biochem. Physiol. 55:55-67. 